\title{
Subfailure damage in ligament: a structural and cellular evaluation
}

\author{
PAOLO P. PROVENZANO,${ }^{1}$ DENNIS HEISEY,${ }^{2}$ KEI HAYASHI,${ }^{3}$ \\ RODERIC LAKES, ${ }^{4}$ AND RAY VANDERBY, JR. ${ }^{1}$ \\ ${ }^{1}$ Department of Biomedical Engineering and Department of Orthopedic Surgery and ${ }^{2}$ Department of \\ Surgery and Department of Biostatistics and Medical Informatics, 53792-3228; ${ }^{3}$ Department of \\ Surgical Sciences, School of Veterinary Medicine, 53706; and ${ }^{4}$ Department of Engineering Physics \\ and Department of Biomedical Engineering, University of Wisconsin, Madison, Wisconsin 53706-1687
}

Received 17 July 2001; accepted in final form 14 August 2001

Provenzano, Paolo P., Dennis Heisey, Kei Hayashi, Roderic Lakes, and Ray Vanderby, Jr. Subfailure damage in ligament: a structural and cellular evaluation. J Appl Physiol 92: 362-371, 2002.-Subfailure damage in ligaments was evaluated macroscopically from a structural perspective (referring to the entire ligament as a structure) and microscopically from a cellular perspective. Freshly harvested rat medial collateral ligaments (MCLs) were used as a model in ex vivo experiments. Ligaments were preloaded with $0.1 \mathrm{~N}$ to establish a consistent point of reference for length (and strain) measurements. Ligament structural damage was characterized by nonrecoverable difference in tissue length after a subfailure stretch. The tissue's mechanical properties (via stress vs. strain curves measured from a preloaded state) after a single subfailure stretch were also evaluated $(n=6$ pairs with a different stretch magnitude applied to each stretched ligament). Regions containing necrotic cells were used to characterize cellular damage after a single stretch. It should be noted that the number of damaged cells was not quantified and the difference between cellular area and area of fluorescence is not known. Structural and cellular damage were represented and compared as functions of subfailure MCL strains. Statistical analysis indicated that the onset of structural damage occurs at $5.14 \%$ strain (referenced from a preloaded length). Subfailure strains above the damage threshold changed the shape of the MCL stress-strain curve by elongating the toe region (i.e., increasing laxity) as well as decreasing the tangential modulus and ultimate stress. Cellular damage was induced at ligament strains significantly below the structural damage threshold. This cellular damage is likely to be part of the natural healing process in mildly sprained ligaments.

cell necrosis; fibroblast; medial collateral ligament; sprain; laxity

MICROTRAUMA OR SUBFAILURE INJURY in tendon and ligament may occur either as the result of overuse or as a single traumatic event (5). Pathologies of the musculoskeletal system, in which microtrauma is thought to play a role, include tendinitis and tendinosis of the upper extremity (20), including tennis elbow (8) and

Address for reprint requests and other correspondence: $R$ Vanderby Jr., Dept. of Orthopedic Surgery, Orthopedic Research Laboratories, 600 Highland Ave., Univ. of Wisconsin, Madison, Madison, WI 53792-3228 (E-mail: vanderby@surgery.wisc.edu). carpal tunnel syndrome (31), as well as lower limb pathologies such as Achilles tendinitis (8) and posterior tibial tendinitis (4), among others. Microtrauma diminishes mechanical properties in knee ligaments (19) and is hypothesized to be a source of lower back pain through microtrauma of either the iliolumbar ligament (35) or the intervertebral disk (42). Fruensgaard and Johannsen (10) reported that partial tears of the anterior cruciate ligament often lead to reduced levels of activity and performance. Although conservative treatment is often successful, tendon and ligament microtrauma and partial tears may accumulate damage to the point that load bearing is compromised and complete rupture or secondary damage occurs (12). In addition, ligament microtrauma may result in increased laxity, which in turn is associated with degenerative joint disease and osteoarthritis (9). These studies suggest the importance of studying subfailure injury and intrinsic healing of ligaments.

A sprain is defined as an acute injury to a ligament or joint capsule without dislocation. Sprains are classified by severity on the basis of clinical examination or imaging. Grade I sprains are mild stretches with no discontinuity of the ligament and no clinically detectable increase in joint laxity. Grade II sprains are moderate stretches in which some fibers are torn. Enough fibers remain intact so that the damaged ligament has not failed. These grade II sprains produce detectable abnormal laxity at the joint compared with the uninjured, contralateral side. Grade III sprains are severe and consist of a complete or nearly complete ligament disruption and result in significant joint laxity. Severe sprains involving complete disruption of the ligament and resulting in significant joint laxity (grade III) constitute $<15 \%$ of all ligament sprains (2). This leaves $>85 \%$ of the sprains in which subfailure damage is the dominant issue.

Early studies of mechanical properties of tendons and ligaments disclosed that irreversible mechanical behavior (and presumably damage) occurs at relatively

The costs of publication of this article were defrayed in part by the payment of page charges. The article must therefore be hereby marked "advertisement" in accordance with 18 U.S.C. Section 1734 solely to indicate this fact. 
low levels of strain [2.5-4.5\% beyond preloaded reference levels $(32,38)]$. In one study of rabbit anterior cruciate ligaments, subfailure injury ( $\sim 80 \%$ of failure stretch) altered the shape of loading curves and thereby increased joint laxity (28). In another study, dramatic reductions in peak force were observed in human cadaveric inferior glenohumeral ligament after repeated subfailure loadings (39). Laws and Walton (23) used a sheep model to investigate a grade II injury to the medial collateral ligament (MCL). They reported an initial 13\% reduction in the tensile strength and an increase in laxity but full recovery at $6 \mathrm{wk}$. They also reported progressive healing by infiltrating fibroblasts with less of the typical inflammatory response seen in complete failure models. The first time interval for histological investigation was after $1 \mathrm{wk}$, so the early healing response was not described. The injury was not well controlled, and the molecular responses in the MCL were not described. Although changes in ligaments have been observed at subfailure loadings, the mechanisms and effects of damage accumulation are still not known.

Cells in the body are subjected to complex mechanical loadings, consisting of tension, compression, shear, or combinations of the three types of load and deformation. Researchers have studied the mechanical stimulation and behavior of cells in vitro $(1,11,13,14$, 40). Hsieh et al. (14) studied human fibroblasts from the anterior cruciate and medial collateral ligaments in vitro under equibiaxial strains of 5 and $7.5 \%$, showing that cell strain induced expression of types I and III collagen. They suggested that remodeling of ligament tissue may take place by a continuous microhealing process whereby scar tissue (predominately type III collagen) is formed and later matures into remodeled tissue. Arnozczky et al. (3) showed that cell deformation increased with tissue strain in situ but that when the relationship is nonlinear and wide, a variation was observed. None of these studies examined cell necrosis in situ or the mechanism by which mechanically induced cell necrosis occurs in soft tissue.

This study examines structural and cellular damage in ligaments after a subfailure damage injury. Sprains are not currently well quantified in this subfailure region. This study 1) quantifies the onset of structural damage in the medial collateral ligament as characterized by nonrecoverable change in tissue length (from preload) after a subfailure stretch, and 2) quantifies regions of cellular damage (not the number of necrotic cells) in ligament as a function of subfailure ligament strain. The tissue's mechanical properties after a subfailure stretch were also evaluated. It should be noted that for this study structural damage is considered nonrecoverable changes in the entire ligament structure and does not reflect examination of the extracellular matrix (ECM) (i.e., collagen fiber or fibril integrity) at the microstructural level.

\section{METHODS}

Experimental studies. This study was approved by the institutional animal use and care committee and meets Na- tional Institutes of Health (NIH) guidelines for animal welfare. Sprague-Dawley male rats (weight $250 \pm 25 \mathrm{~g}$ ) were used as our animal model. Immediately after death, all extraneous tissue was carefully dissected to expose each MCL. Intact MCLs, including femoral and tibial bone segments, were carefully harvested with care taken not to disturb the ligament insertion sites. The tissues were kept hydrated in Hanks' physiological solution $(\mathrm{pH}=7.4)$. Structural damage ( $n=25$ ligaments $)$ and cellular damage $(n=24$ ligaments $)$ were evaluated in this study. Twelve additional ligaments ( $n=6$ pairs) were used to evaluate changes in the ligaments' mechanical properties after a subfailure stretch.

To initiate a subfailure stretch in the MCL, tissues were placed into a custom-designed load frame with special structures to hold the femur and tibial end sections of the sample in an anatomic position that loads the fibers as uniformly as possible. Engineering strain $(\epsilon)$ was measured by placing graphite-impregnated silicon grease markers on the specimen and using video dimensional analysis to measure displacement. The experimental system (load frame, camera, and image processor) has a resolution of at least $10 \mu \mathrm{m}$ and repeated measurements at a fixed length of optical markers are consistently reproducible to at least $10 \mu \mathrm{m}$. Force was measured with a transducer having a resolution $\sim 0.0025 \%$ of the maximum force. Data were acquired on a personal computer with Labtech Notebook (Laboratory Technologies, Wilmington, MA) and recorded on video to synchronize force and displacement data. NIH Image (25) was used to capture and analyze individual frames of the videotaped tests. The $x-y$ coordinate center (centroid coordinates) of each marker was used to calculate the distance between the silicon markers and thus ligament displacement after loading. For the structural damage group $(n=25)$, the tissue was preconditioned (10 cycles at $1 \%$ strain) and allowed to recover for $10 \mathrm{~min}$. After preconditioning and recovery (to reduce a viscoelastic creep-recovery response), the gauge length $\left(L_{\mathrm{O}}\right)$ was established after loading of the specimens with $0.1 \mathrm{~N}$. Preconditioning and preload were included in the experimental protocol to obtain a uniform zero point and to allow the sample to settle into the testing apparatus. After preload, the tissue was subjected to a subfailure stretch in displacement control at a strain rate of $10 \% / \mathrm{s}$. Tissues that were subjected to zero stretch ( $0 \%$ controls) were harvested and hydrated as stated above and placed in the load frame with markers in the same preloaded fashion as the subfailure stretched ligaments with length measured at each time point. The level of subfailure stretch not resulting in grade III ligament failure applied to each ligament varied between 0 (no stretch) and stretch $\sim 12 \%$ based on preliminary studies in this laboratory. If a grade III ligament injury occurred (tissue failure displaying an abrupt drop in force), the ligament was excluded from the study. It should be noted that some grade III failures occurred at slightly lower strains than $12 \%$ whereas some failed at larger strain; thus $\sim 12 \%$ strain was the highest subfailure stretch we were able to attain. After the subfailure stretch, the tissue was unloaded and allowed to recover for 10 min $[>300 \times$ the length of loading during the test; at least $10 \times$ the length of loading is recommended by Turner (37)], in an attempt to ensure that changes in length were not the result of a viscoelastic creep-recovery response. Viscoelastic experiments on rat MCLs subjected to low loads or displacements below $5 \%$ tissue strain performed in this laboratory have shown that a recovery time equal to $10 \times$ the test time allows the rat MCL to return to the initial $L_{\mathrm{O}}$ at preload (30). After tissue recovery, the length $\left(L_{\mathrm{S}}\right)$ of the MCL was measured at the preload level of $0.1 \mathrm{~N}$. The difference in nonrecoverable length (laxity) is defined as structural damage for 
this study. The length difference was normalized by the original length $\left(L_{\mathrm{O}}\right)$ and is expressed as a percentage: $\mathrm{D}_{\mathrm{S}}=$ $100 \cdot\left[\left(L_{\mathrm{S}}-L_{\mathrm{O}}\right) / L_{\mathrm{O}}\right]$, where $\mathrm{D}_{\mathrm{S}}$ is a measure of structural damage. It should be noted that the symbol $D_{S}$ in this study differs from the use of the symbol $\mathrm{D}$ as the damage variable in continuum damage mechanics $(6,21)$.

To demonstrate the effect of a damage-inducing subfailure stretch on the mechanical properties of the ligament, stressstrain curves for six additional pairs of ligaments $(n=12$ ligaments) were examined. Paired ligaments from each animal were used with one MCL as a control, i.e., not receiving a subfailure stretch before being pulled to failure, and the contralateral ligament receiving a subfailure stretch before being pulled to failure. The assumption of symmetry in contralateral knee ligaments, used herein, has been validated (7). The cross-sectional area was calculated by measuring the width and thickness (10- $\mu \mathrm{m}$ resolution) of the ligament and assuming an elliptical cross section. The ligaments were preconditioned at 10 cycles of $1 \%$ strain, allowed to recover, and stretched in displacement control at 10\%/s. Each subfailure-stretched ligament received a different magnitude of subfailure stretch, ranging from 0 to $9 \%$. The six ligaments receiving a subfailure stretch were loaded, allowed time to recover viscoelastic deformations as above, and then pulled to failure at $10 \% / \mathrm{s}$. The mechanical properties of the ligaments that received subfailure stretches were compared with the mechanical properties of the contralateral control ligament.

Tissues evaluated for cellular damage $(n=24)$ were stretched in the same fashion as the structural damage group. Confocal microscopy ( $n=22$ ligaments) with a cell viability assay detected live and necrotic cells (mainly fibroblasts in ligament). Tissues were prepared for confocal microscopy immediately after tissue stretch, and the time from stretch to viewing of the tissues was $\sim 1 \mathrm{~h}$, during which staining was performed. By use of a technique similar to that used in Ohlendorf et al. (27), in situ staining was done with calcein and ethidium homodimer, whereby live cells metabolize calcein and show green fluorescence and membranes of necrotic cells are penetrated by ethidium homodimer, which results in red fluorescent staining of the nuclei. Image scans through the depth of the tissue were compiled and overlaid. Yellow areas on the images are a summation of both live and necrotic cells and were counted as regions of cellular damage. Black sections indicated that no stained cells were present in that region. Tissues that were subjected to zero stretch were harvested from the rat, as stated above, and placed in the load frame with markers in the same fashion as the subfailure stretched ligaments, preloaded, and then processed as $0 \%$ strain baselines. Images of the ligaments from confocal microscopy were stored digitally, and regions containing necrotic cells were quantified with NIH Image (25). The number of necrotic cells was not counted and is not quantified in this study because the relationship between fluorescence area (present in the images) and cell area is not known and scans are overlaid. The colors from red to yellow to green that are present in the ligament were ordered and assigned an index. A constant threshold was set as the boundary between yellow (regions with some necrotic cells) and green (regions where cells had intact membranes). The number of pixels of green was used to quantify the regions of the ligament with viable cells, and the yellow and red pixels were used to quantify the regions of the ligament with necrotic cells. The background index (black in color) of the image was identified and pixels of that index were eliminated, so that the nonred, nonyellow, and nongreen pixels were not counted. The area of the ligament was measured in units of pixels and used to normalize the regions containing necrotic cells. Hence, the measure of cellular damage $\left(D_{C}\right)$ is a nondimensional unit represented as a percentage of the form $D_{C}=100 \cdot$ (regions containing necrotic cells/tissue area) (pixels/pixels).

In addition, transmission electron microscopy (TEM) $(n=$ 2 ligaments) was performed on control $(\epsilon=0 \%)$ and stretched $(\epsilon=3.2 \%)$ ligament to view the cells. The specimens were fixed in modified Karnovsky's solution, postfixed in 1\% osmium tetroxide, and stained with $1 \%$ uranyl. After sequential dehydration in ethanol and infiltration in Epon-Araldite and propylene oxide, specimens were embedded in 100\% Epon-Araldite and polymerized at $60^{\circ} \mathrm{C}$. Ultrathin $(70 \mathrm{~nm})$ sections were cut, placed on grids, stained with lead citrate, and viewed by using a transmission electron microscope.

Statistical analysis. Statistical analysis was performed to determine the strain at which the onset of damage occurs from structural and cellular standpoints and to determine whether the two were different from one another. An observation of cellular damage (cellular damage per ligament) was defined as $\mathrm{D}_{\mathrm{ci}}$. The general expression $\mathrm{D}_{\mathrm{ci}} \sim \mathrm{N}\left(\mu_{\mathrm{ci}}, \sigma_{\mathrm{c}}^{2}\right)$ indicates that $D_{\text {ci }}$ was expected to have the mean value $\mu_{\text {ci }}$, a normal distribution about this mean value with variance $\sigma_{\mathrm{c}}^{2}$. The mean value was modeled as a function of strain $\epsilon_{\mathrm{ci}}$ as

$$
\mu_{\mathrm{ci}}=\beta_{\mathrm{c}}\left(\varepsilon_{\mathrm{ci}}-\theta_{\mathrm{c}}\right) \mathrm{I}_{\mathrm{c}}\left[\varepsilon_{\mathrm{ci}}\right]
$$

with

$$
\begin{gathered}
\mathrm{I}_{\mathrm{c}}\left(\varepsilon_{\mathrm{ci}}\right)=0 \quad \text { for } \quad \varepsilon_{\mathrm{ci}}<\theta_{\mathrm{c}} \\
\mathrm{I}_{\mathrm{ci}}\left(\varepsilon_{\mathrm{ci}}\right)=1 \quad \text { for } \quad \varepsilon_{\mathrm{ci}} \geq \theta_{\mathrm{c}}
\end{gathered}
$$

where $\theta_{\mathrm{c}}$ was the strain threshold below which cellular damage is zero and above which cellular damage increases linearly with strain with slope $\beta_{\mathrm{c}}$. The kink at $\theta_{\mathrm{c}}$ was obtained by the indicator function $\mathrm{I}_{\mathrm{c}}\left(\epsilon_{\mathrm{ci}}\right)$, which equals 0 when $\epsilon_{\mathrm{ci}}<\theta_{\mathrm{c}}$ and equals 1 otherwise. A similar development was applied for structural damage $D_{\mathrm{si}}$, modeling the mean value $\left(\mu_{\mathrm{si}}\right)$ as a function of strain $\left(\epsilon_{\mathrm{si}}\right)$, hence

$$
\mu_{\mathrm{si}}=\beta_{\mathrm{s}}\left(\varepsilon_{\mathrm{si}}-\theta_{\mathrm{s}}\right) \mathrm{I}_{\mathrm{s}}\left(\varepsilon_{\mathrm{si}}\right)
$$

with

$$
\begin{array}{lll}
\mathrm{I}_{\mathrm{s}}\left[\varepsilon_{\mathrm{si}}\right]=0 & \text { for } \quad \varepsilon_{\mathrm{si}}<\theta_{\mathrm{s}} \\
\mathrm{I}_{\mathrm{s}}\left[\varepsilon_{\mathrm{si}}\right]=1 & \text { for } \quad \varepsilon_{\mathrm{si}} \geq \theta_{\mathrm{s}}
\end{array}
$$

To obtain the "best" estimates of the parameters $\beta_{\mathrm{c}}, \theta_{\mathrm{c}}, \beta_{\mathrm{s}}$, and $\theta_{\mathrm{s}}$ (estimates of the nuisance parameters $\sigma_{\mathrm{c}}$ and $\sigma_{\mathrm{s}}$ were also needed), maximum likelihood estimates (MLEs) were used (e.g., Ref. 24). MLEs are those values of the parameters that maximize the probability of observed data. Thus, to obtain MLEs, the probability density function of the observed data was constructed; this probability density function is referred to as the likelihood equation. For mathematical convenience, rather than maximizing the likelihood directly, the log likelihood was maximized (the position of maxima are invariant to log transformation).

For the $n_{\mathrm{c}}$ cellular damage samples, the observations were assumed to be independent and normally distributed. Hence, the log likelihood for the cellular data was expressed as

$$
\left.\mathrm{L}_{\mathrm{c}}=-\left(n_{\mathrm{c}} / 2\right) \ln \left(2 \pi \sigma_{\mathrm{c}}^{2}\right)-\mathrm{S}_{\mathrm{c}}^{2} / 2 \sigma_{\mathrm{c}}^{2}\right)
$$

where $\mathrm{S}_{\mathrm{c}}^{2}$ represented the cellular damage sum of squares. This defined the sum of squared errors about the mean values, or

$$
\mathrm{S}_{\mathrm{c}}^{2}=\sum_{i=1}^{n_{\mathrm{c}}}\left(y_{\mathrm{ci}}-\mu_{\mathrm{c}}\right)^{2}
$$


or more specifically,

$$
\mathrm{S}_{\mathrm{c}}^{2}=\sum_{i=1}^{n_{\mathrm{c}}}\left(y_{\mathrm{ci}}-\beta_{\mathrm{c}}\left(x_{\mathrm{ci}}-\theta_{\mathrm{c}}\right) I_{\mathrm{c}}\left[x_{\mathrm{ci}}\right]\right)^{2}
$$

A similar expression for the log likelihood of structural damage $\left(\mathrm{L}_{\mathrm{s}}\right)$ was written. Because of the additive nature of log likelihoods, the log likelihood for all of the observed data, cellular and structural combined, was defined as $\mathrm{L}=\mathrm{L}_{\mathrm{c}}+\mathrm{L}_{\mathrm{s}}$. Then $\mathrm{L}$ was maximized with respect to the six parameters $\left(\beta_{\mathrm{c}}, \theta_{\mathrm{c}}, \beta_{\mathrm{s}}, \theta_{\mathrm{s}}, \sigma_{\mathrm{c}}, \sigma_{\mathrm{s}}\right)$. This was done by taking the first partial derivatives, setting them to 0 , and solving for the parameters. This was conveniently done in two steps. The first step was to maximize L just with respect to $\sigma_{\mathrm{c}}$ and $\sigma_{\mathrm{s}}$. Because the solution to $\partial \mathrm{L} / \partial \sigma_{\mathrm{c}}=0$ is $\sigma_{\mathrm{c}}^{2}=\mathrm{S}_{\mathrm{c}}^{2} / n_{\mathrm{c}}, \sigma_{\mathrm{c}}$ was eliminated from $\mathrm{L}_{\mathrm{c}}$ by substituting $\sigma_{\mathrm{c}}^{* 2}$, yielding

$$
\mathrm{L}_{\mathrm{c}}^{*}=-\left(n_{\mathrm{c}} / 2\right)\left[\ln \left(2 \pi \sigma_{\mathrm{c}}^{* 2}\right)-1\right]
$$

the so-called cellular profile log likelihood. The cellular profile log likelihood (i.e., the cellular log likelihood maximized with respect to $\sigma_{c}$ ) became a function of $\beta_{c}$ and $\theta_{c}$. Thus maximizing the total profile $\log$ likelihood $\mathrm{L}^{*}=\mathrm{L}_{\mathrm{c}}^{*}+\mathrm{L}_{\mathrm{s}}^{*}$ with respect to just the four parameters $\left(\beta_{c}, \theta_{c}, \beta_{s}, \theta_{s}\right)$ was equivalent to maximizing the total log likelihood with respect to all six. For any fixed $\theta_{\mathrm{c}}$ and $\theta_{\mathrm{s}}, \mathrm{L}^{*}$ was maximized by individually finding the least-squares estimates of $\beta_{c}$ and $\beta_{s}$, which individually maximized the components $\mathrm{L}_{c}^{*}$ and $\mathrm{L}_{\mathrm{s}}^{*}$.

A goal of this study was to determine whether the threshold for cellular damage was different from that for structural damage. That is, a difference (i.e., rejecting the null hypothesis $H_{0}: \theta_{c}=\theta_{\mathrm{s}}$ in favor of the alternative hypothesis $\mathrm{H}_{\mathrm{A}}: \theta_{\mathrm{c}} \neq$ $\left.\theta_{\mathrm{s}}\right)$ was sought with a likelihood ratio test. The maximum log likelihood obtained under the alternative model was defined as $\mathrm{L}^{\mathrm{A}}$; this was simply the maximum of the log likelihood described above with all six parameters free to vary. The maximum log likelihood obtained under the constrained null hypothesis model where $\theta_{c}=\theta_{\mathrm{s}}$ was denoted as $\mathrm{L}^{0}$. Then, $\chi^{2}=2\left(\mathrm{~L}^{\mathrm{A}}-\mathrm{L}^{0}\right)$ had a $\chi^{2}$ distribution with one degree of freedom under the null distribution.

A line search was used to find the value of $\theta$ that maximized the null hypothesis log likelihood, $\mathrm{L}^{0}$. To start, $\theta$ was set to 0 . Then, conditional on this $\theta$, the values of $\beta_{\mathrm{c}}$ and $\beta_{\mathrm{s}}$ that maximize $L^{*}$ were found by least squares. Then $\theta$ was incremented by a small amount, and the process was repeated. $\mathrm{L}^{0}$ was the largest value of $\mathrm{L}^{*}$ observed, and the MLEs under the null hypothesis were the corresponding estimates for $\beta_{\mathrm{c}}, \beta_{\mathrm{s}}, \theta, \sigma_{\mathrm{c}}$, and $\sigma_{\mathrm{s}}$. A similar process was used to maximize the alternative hypothesis log likelihood to obtain $\mathrm{L}^{\mathrm{A}}$. Because of the additive nature of the log likelihood, $\mathrm{L}_{\mathrm{c}}^{*}$ and $\mathrm{L}_{\mathrm{s}}^{*}$ were maximized separately by individual line searches.

Confidence intervals about the parameter estimates were constructed with the profile likelihood method. Let $\mathrm{L}^{\mathrm{p}}$ be $\mathrm{L}^{*}$ maximized for all parameters but $\theta_{\mathrm{s}}$, a so-called profile likelihood. Ninety-five percent profile likelihood confidence intervals for $\theta_{\mathrm{s}}$ were constructed by finding the values of $\theta_{\mathrm{s}}$ that satisfy the equality $3.84=2\left(\mathrm{~L}^{\mathrm{A}}-\mathrm{L}^{\mathrm{p}}\right)$, where 3.84 is the 95 th percentile of the $\chi^{2}$ distribution.

\section{RESULTS}

Results indicate that tissue stretch-induced structural damage and cellular damage are fundamentally different in their behaviors when analyzed as a function of applied strain. Qualitatively, regions of cellular damage can be seen to increase with strain by exami- nation of the confocal microscopic images (Fig. 1). In addition, the strain produced extensive damage to individual cells as seen in TEM images at 3.2\% ligament strain (Fig. 2). These images show a typical cell for which the membrane is intact and the cellular contents

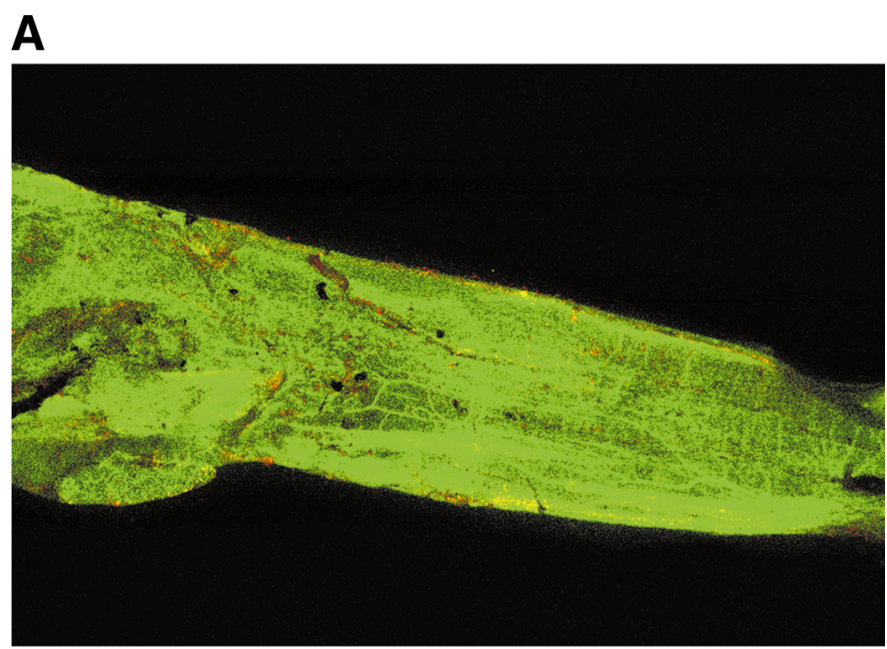

B

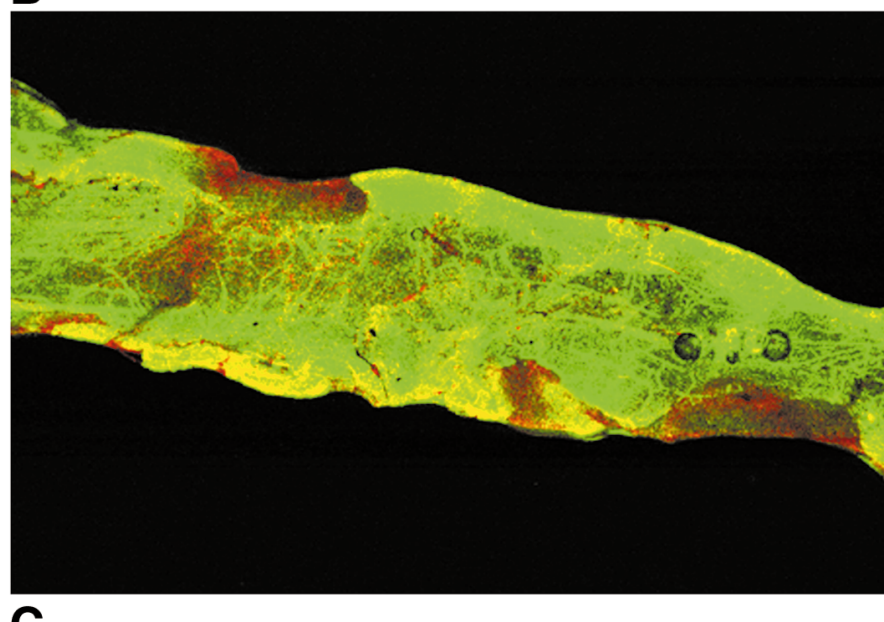

C

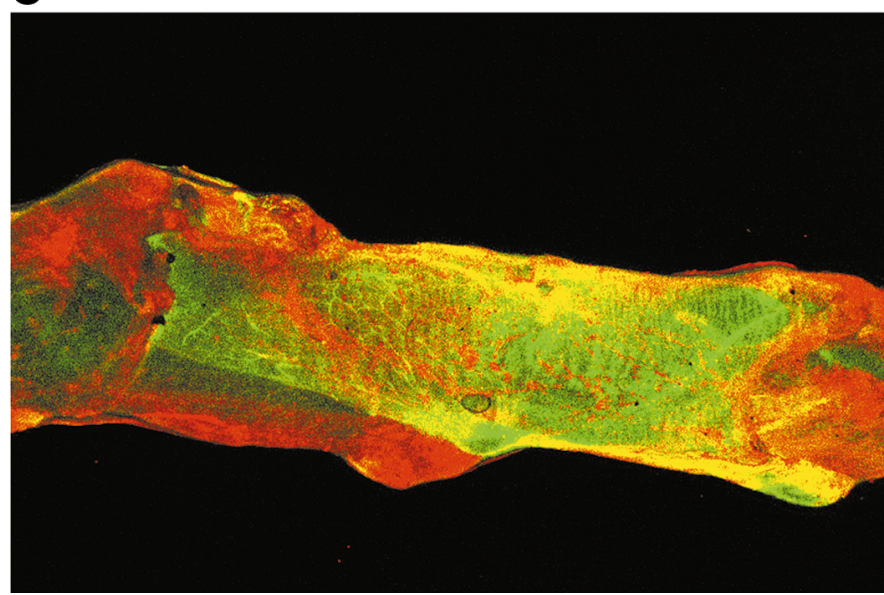

Fig. 1. Rat medial collateral ligaments at strains of $0(A), 6(B)$, and $11 \%(C)$. Red and yellow regions indicate cell necrosis, and green regions are healthy live cells. Note the extent of necrosis in the strained ligaments and the increase in necrosis with higher strains. 
Fig. 2. Fibroblasts in a normal unstrained ligament (left) and fibroblasts in a strained $(\epsilon=3.2 \%)$ ligament (right). Normal ligaments have intact cellular membranes in a longitudinal section at $24,000 \times$ (top left), transverse section at $9,100 \times$ (middle left), and longitudinal section at $24,000 \times$ (bottom left). Ligament that has been strained to $3.2 \%$ (right) shows cellular damage in the form of ruptured cell membranes and the release of cellular contents in the extracellular matrix in a longitudinal section at $24,000 \times($ top right), transverse section at $9,100 \times$ (middle right), and longitudinal section at $24,000 \times$ (bottom right). Note interdigitation of collagen fibrils with normal ligaments in left column.
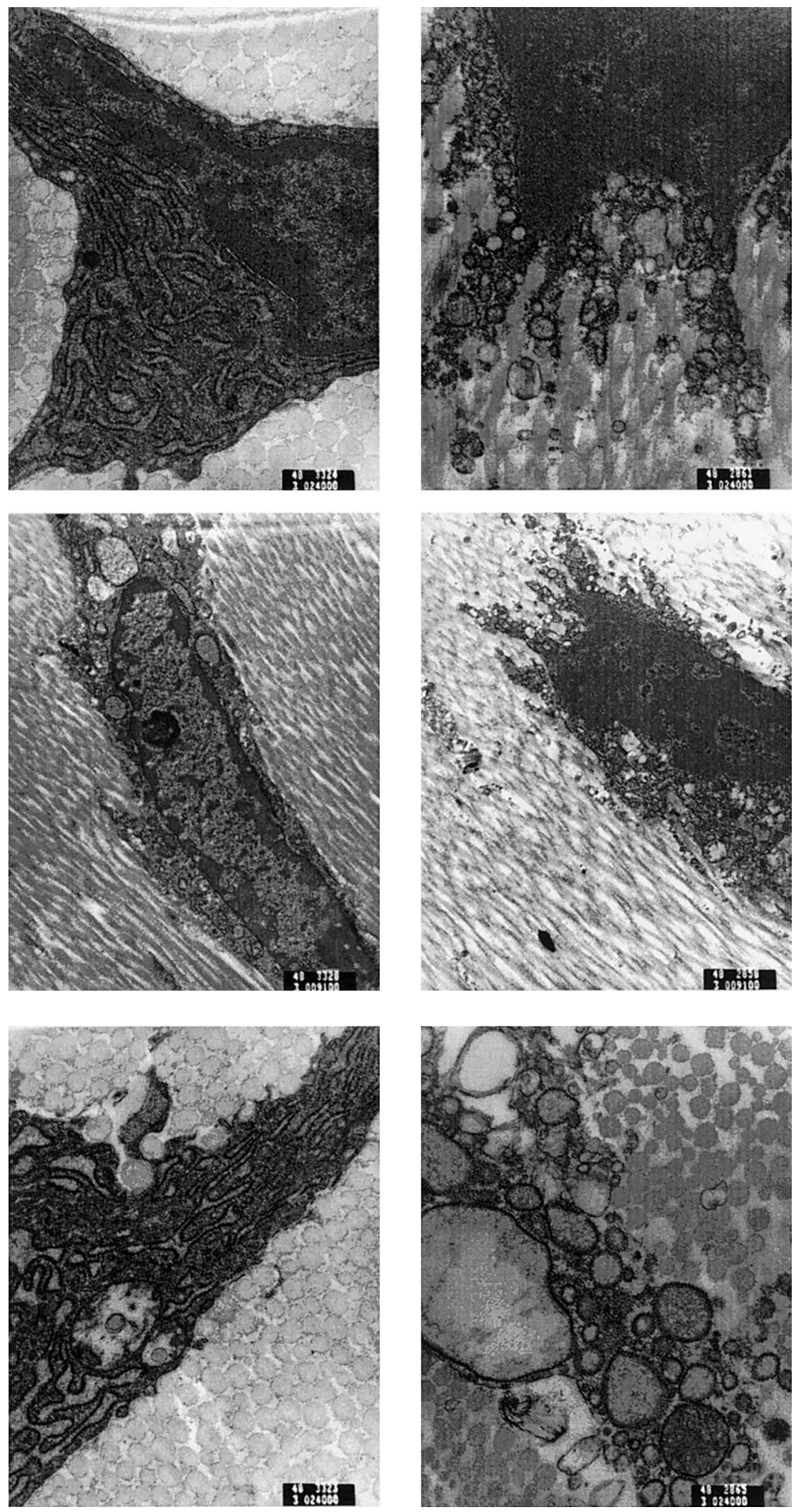

are undisturbed in a ligament with $0 \%$ strain. A typical mechanically damaged cell in the strained ligament displays a cell membrane that has been ruptured and the cellular contents that have been released into the
ECM. Data for structural damage $\left(\mathrm{D}_{\mathrm{S}}\right)$ and cellular damage $\left(D_{C}\right)$ seen in Figs. 3 and 4, respectively, clearly show the different strain-dependent behaviors for structural and cellular damage. Statistically, the onset 


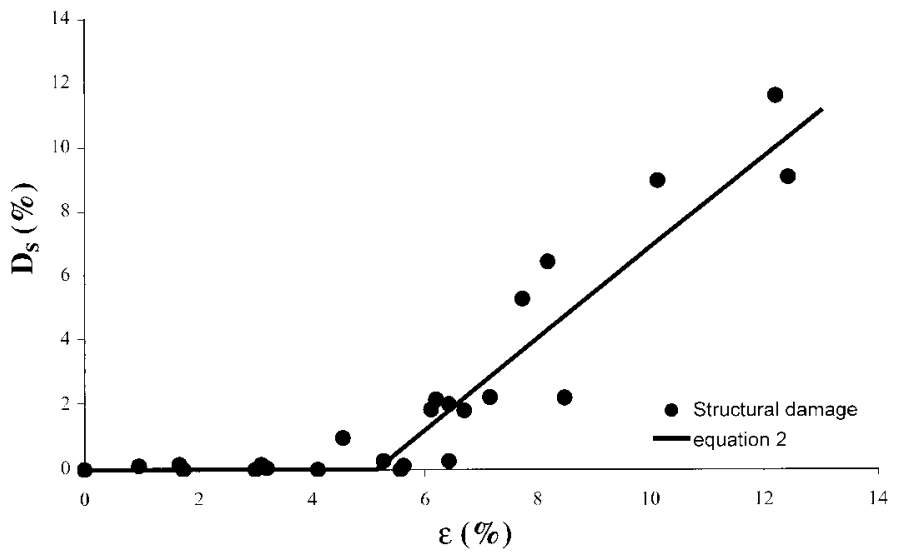

Fig. 3. Structural damage $\left(D_{S}\right)$ vs. strain $(\epsilon)$ in rat medial collateral ligament. Structural damage represents tissue laxity. The onset of damage is at $5.14 \%$ strain, as can be seen by $E q .2$. Note that this level of strain is well into the linear region of the stress-strain curve for this tissue, as can be seen from Fig. 5 .

of structural damage $\left(\theta_{\mathrm{s}}\right)$ was found to be at a strain of $5.14 \%$ from preload, and this level of strain can be seen to be well within the linear region of the stress-strain curve for the rat ligaments used in this study (Figs. 5). The $95 \%$ profile likelihood confidence intervals for structural damage are 4.50-5.69. The onset of changes in cellular damage $\left(\theta_{c}\right)$ was found to be $0(\epsilon=0 \%)$. That is, statistically, changes in cellular damage in rat MCLs begin with the application of ligament strain from preload, and structural damage occurs at strains $>5.14 \%$ from preload. Some regions of cellular damage can be seen at $0 \%$ strain (i.e., preloaded treatment), yet statistical analysis could not reveal any other onset because of scatter in the data. No consistent localization of regions of cellular damage could be identified in the ligaments. The slope values $(\beta)$ for structural and cellular damage are 1.42 and 4.00, respectively. From these values of $\theta$ and $\beta, E q s .1$ and 2 are

$$
\begin{aligned}
& \mu_{\mathrm{ci}}=4.00\left(\varepsilon_{\mathrm{ci}}-0.00\right) \mathrm{I}_{\mathrm{c}}\left(\varepsilon_{\mathrm{ci}}\right) \\
& \mu_{\mathrm{si}}=1.42\left(\varepsilon_{\mathrm{si}}-5.14\right) \mathrm{I}_{\mathrm{s}}\left(\varepsilon_{\mathrm{si}}\right)
\end{aligned}
$$

with the indicator function $\mathrm{I}\left(\epsilon_{\mathrm{i}}\right)$ equal to 0 when $\epsilon_{i}<\theta$ and equal to 1 otherwise (Fig. 3). The null hypothesis of identical thresholds has $\mathrm{H}_{0}: \theta_{\mathrm{c}}=\theta_{\mathrm{s}}$, and the alternative hypothesis is $H_{\mathrm{A}}: \theta_{\mathrm{c}} \neq \theta_{\mathrm{s}}$. Performing a line search finds the maximum likelihood under the null hypothesis to be $L^{0}=-127.152$ ( $\theta$ estimated to be 4.79$)$. Performing a grid search yields $L^{\mathrm{A}}=-109.5\left(\theta_{\mathrm{c}}\right.$ estimated at $0, \theta_{\mathrm{s}}$ estimated at 5.14). The equation $\chi^{2}=2\left(\mathrm{~L}^{\mathrm{A}}-\mathrm{L}^{0}\right)$ would have a $\chi^{2}$ distribution with one degree of freedom under the null distribution. With $\chi^{2}=35.3$, the null hypothesis is soundly rejected $(P<0.0001)$ and the alternate hypothesis accepted, indicating that the onset of structural and cellular damage occur at significantly different levels of strain. Stress-strain curves demonstrate no change in shape when the subfailure stretch is below the damage threshold (Fig. 5, A-C). A subfailure stretch above the damage threshold elongates the toe region of the stress-strain curve and decreases tissue stiffness and ultimate stress (Fig. 5, $D-F)$.

\section{DISCUSSION}

Subfailure damage has been shown to alter the mechanical properties of the anterior cruciate ligament and lengthen the toe region of the force-displacement curve, which results in increased joint laxity (28). Results from this study indicate that rat medial collateral ligaments strained above 5.14\% from preload do not regain their original length after significant recovery time $(300 \times$ the time of test). This recovery is considerably longer than other studies showing that ligaments stretched below 5\% completely recover in $<10 \times$ the time of test (30). Hence, we conclude that ligaments stretched beyond this threshold remain "stretched." These findings are valuable to researchers performing multiple tests on the same ligament specimen because no change in length or properties is evident in the tissue below $\sim 5 \%$ when testing under the methods described in this study for rat.

We speculate that the increase in elongation after $\sim 5 \%$ strain and change in mechanical properties are the result of fiber damage arising from two possible mechanisms. One mechanism would be torn or plastically deformed fibers. Torn fibers would be consistent with the fiber failure mode of Hurschler's micromechanical model for ligament behavior (16), and plastically deformed fibers could be supported by experimental observations by Sasaki et al. $(33,34)$ and Kukreti and Belkoff (22) who observed that collagen fibrils, which make up collagen fibers, elongate during tendon loading. In addition, Yahia et al. (41), using scanning electron microscopy, reported damage to collagen fibers in subfailure strained ligaments. Another possible mechanism for the observed ligament laxity could be biochemical degradation of the ECM from protease release associated with the observed cellular necrosis. Regardless of the mechanism, the resulting increase in tissue length represents tissue laxity and can be hypothesized to increase joint laxity.

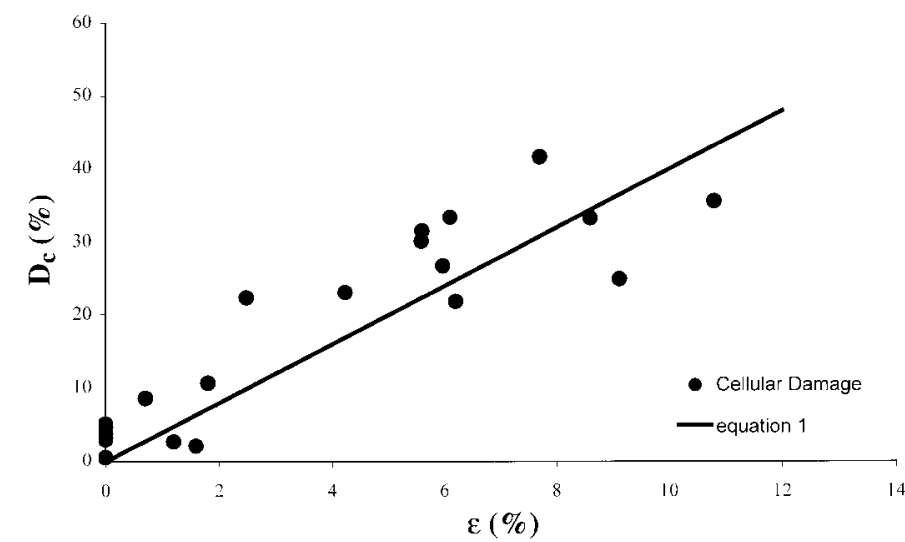

Fig. 4. Cellular damage $\left(\mathrm{D}_{\mathrm{C}}\right)$ vs. strain $(\epsilon)$ in the rat medial collateral ligament. Necrotic area is normalized by the area of the ligament. Equation 1 demonstrates that cellular damage statistically begins with the onset of loading (from a preloaded state) and occurs at lower strain levels than structural damage. 
A
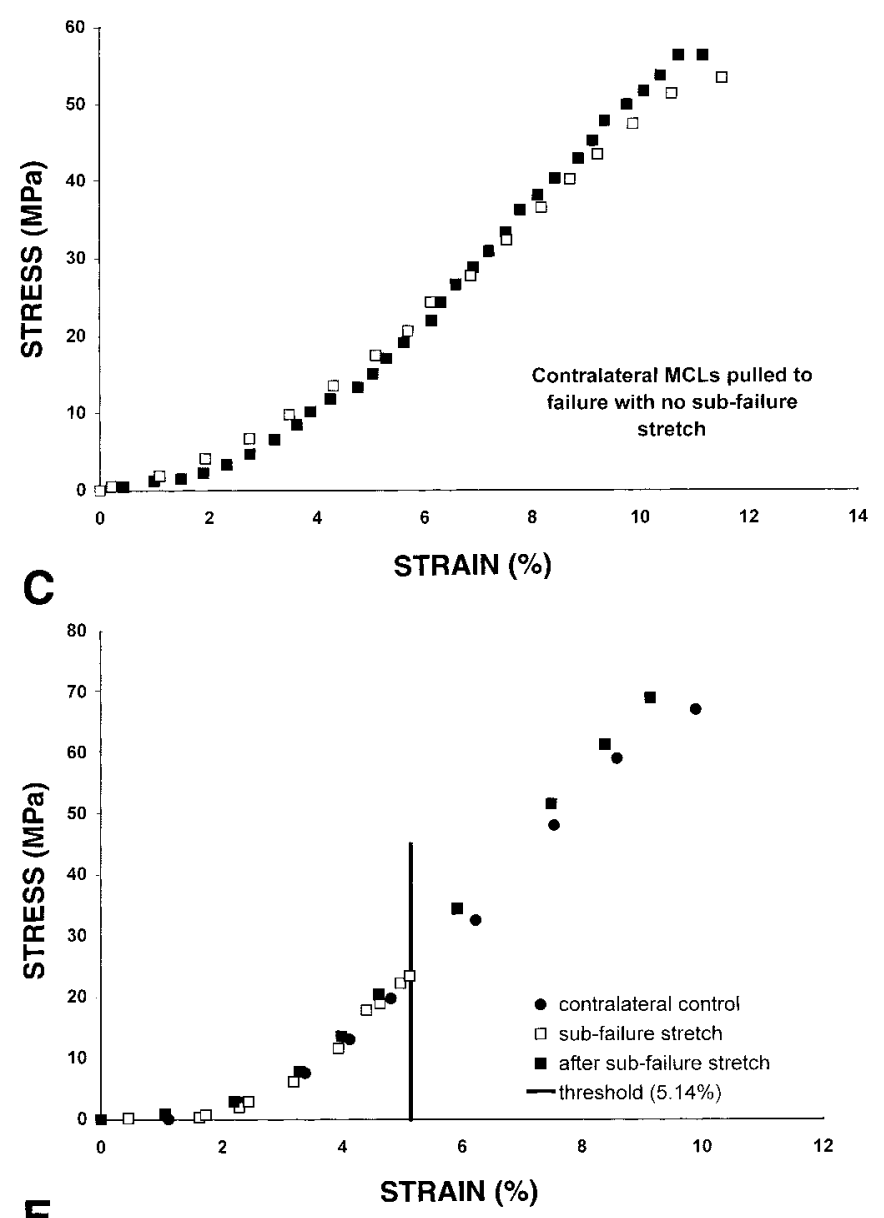

E

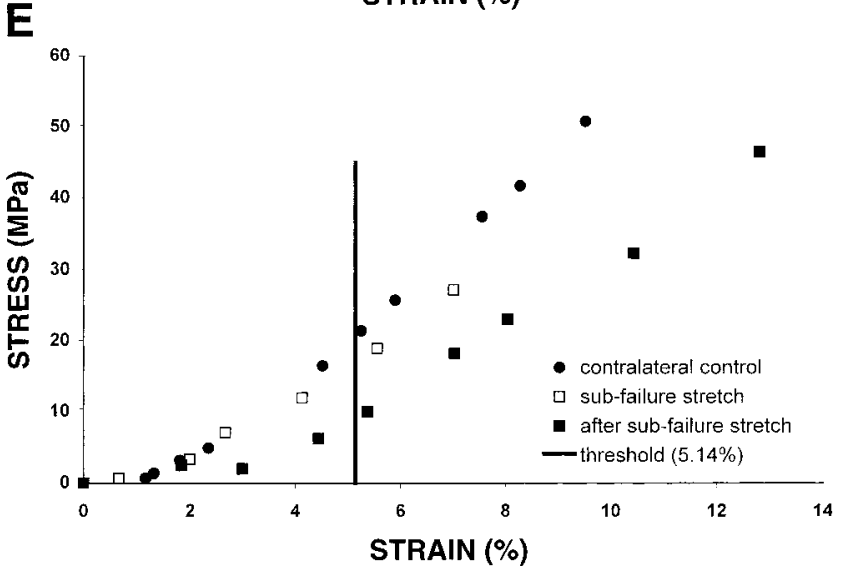

B
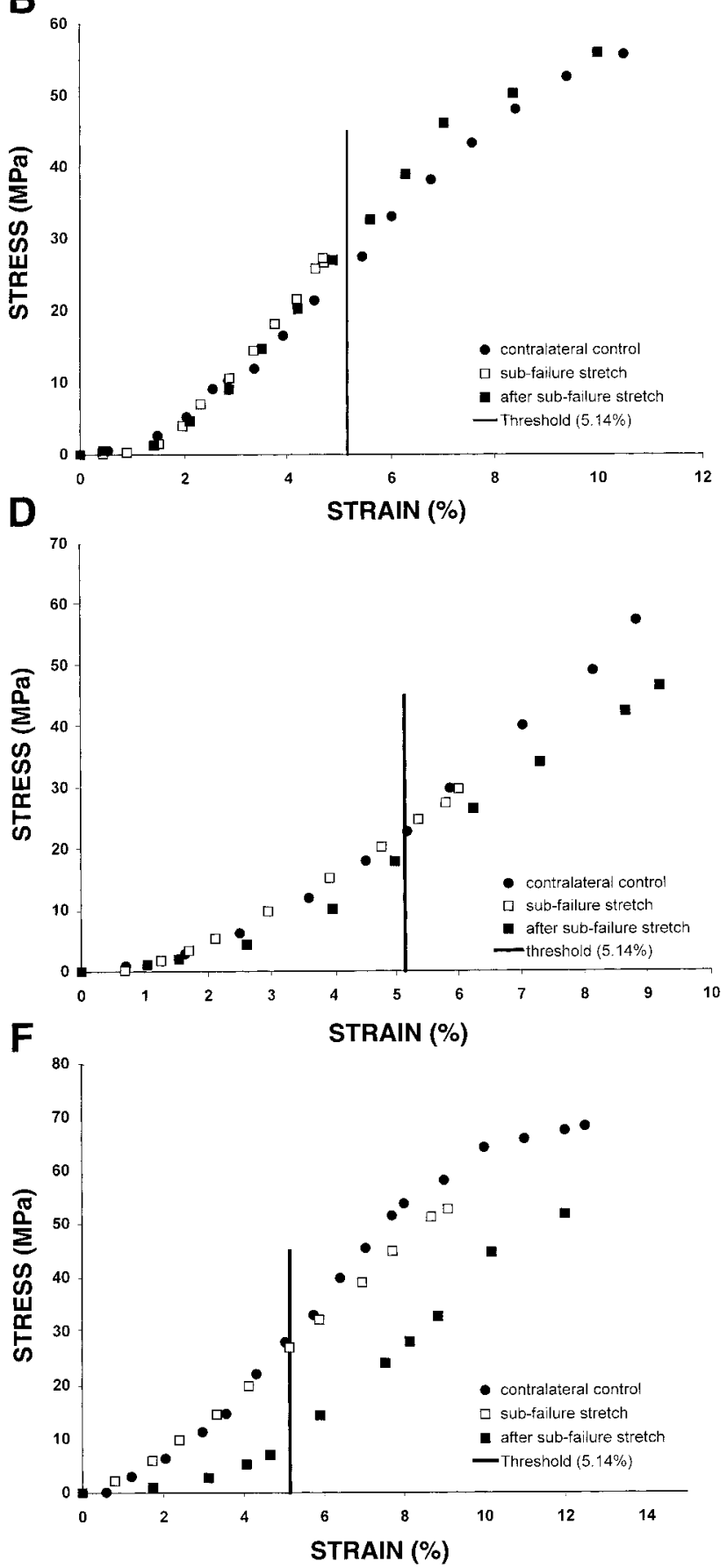

Fig. 5. Stress-strain curves for a control rat medial collateral ligament $(\bullet)$, subfailure stretch $(\square)$, and stretched ligament ( $\mathbf{\square})$ that received a subfailure stretch. The subfailure stretch reached a peak strain of $0 \%(A), 4.7 \%(B)$, $5.1 \%(C), 6 \%(D), 7 \%(E)$, and $9 \%(F)$. Subfailure strains $(\square)$ below $5.14 \%(A-C)$ did not change the toe region or tangential modulus on a subsequent stretch ( $\mathbf{a})$, and the toe region, tangential modulus, and ultimate stress of the ligament $(\square)$ were not changed compared with the contralateral normal ligament $(\bullet)$. Subfailure strains $(\square)$ above $5.14 \%(D-F)$ elongated the toe region and lowered the tangential modulus ( $\mathbf{\square})$ compared with the initial stretch ( $\square$ ). The toe region was elongated and the tangential modulus and ultimate stress decreased ( $\mathbf{a})$ compared with the contralateral normal ligament $(\bullet)$. Note the relative symmetry in contralateral ligaments in $0 \%$ subfailure strain $(A)$.

The statistical threshold for structural damage is well into the linear region of the stress-strain curve, a region unlikely to be regularly reached in normal activity. The upper half of the linear region of stress- strain curve has been associated with partial tearing of anterior cruciate ligaments (26). A microstructural study of the rat MCL in situ using scanning electron microscopy has shown that during knee flexion normal 
ligament has a crimped pattern in knee flexion that is only removed near full extension (17). Our preload removes some crimping and hence results in our preloaded reference length being longer than typical reference lengths in vivo.

The statistical threshold of cellular damage was found to be at $0 \%$ strain from preload in the rat MCL. That is, statistically, cellular damage begins with the application of tissue strain. It should be noted that physically one would not expect an increase in cellular damage at small strains as our statistical analysis implies. However, necrotic cells are present in the control tissues $(\epsilon=0$, i.e., preload) and are present after very small strains (above reference preload). This behavior did not allow the authors to identify any statistical threshold other than the preloaded value. One would not expect high levels of cell necrosis during normal activity; therefore, as mentioned in the previous paragraph, the strains represented when this method is used are likely to be higher than those typically seen in the rat in vivo. The ratio of the area of fluorescence to the actual cell dimensions is not known, and therefore the number of necrotic cells is not counted nor is the percent area of necrotic cells in the tissue quantified. In addition, cellular changes may take place during the processing of the tissues because of processing time and handling, elevating the magnitude of cellular damage. However, all tissues were prepared in the same controlled manner, and changes in cellular damage were seen between preloaded zero strain controls and stretched ligaments, with increasing cellular damage with increasing strain indicating changes relative to the reference values. This difference in damage properties indicates fundamentally different behaviors between cellular and structural damage.

This study quantifies regions of cellular damage in the ligament as a function of strain. As stated in the previous paragraph, this work does not count the number of necrotic cells in the ligament. To the authors' knowledge, no other study has quantified cellular damage in ligament as a function of strain. However, mechanical stimulation of cells in vitro has been studied $(1,11,13,14,40)$. Strain in fibroblasts during in vitro equibiaxial testing on membranes are often higher than the tissue strains at which we are reporting cell damage $(\epsilon>2 \%$ from a preloaded state). However, the relationship between reference (initial) strains used in these studies compared with the complex cell loading in an in vivo state is unknown. Furthermore, differences between the reference strain in our study and previously published in vitro cell deformation studies is also unknown.

We propose that microstructural irregularities in ECM organization create local distortions in fibroblasts during ligament strain that result in the cellular damage reported in this study. Fibroblasts in connective tissue are found to be in columns between fibril bundles $(18,36)$, and fibrils cross and interweave (29). Using confocal microscopy to track cells as a measure of microstructural strain during tendon tissue elonga- tion, Hurschler et al. (15) reported that microstructural strains in ligament can be both larger and smaller than macroscopic ligament strain $( \pm \sim 2 \%$ of the ligament strain) at different locations in the same tissue and that, at the microstructural level, even negative strains can exist. Because fibroblast cells adhere to ECM collagen fibrils, cell deformation is related to fibril displacement and deformation. Squier and Bausch (36) reported junctional attachments between processes of different and same fibroblasts in rat tail tendon. In addition, Squier and Bausch (36) showed that fibroblasts exhibited invaginations of single or groups of collagen fibrils and stated that "this arrangement may be indicative of fibril elongation or serve to transmit tension between the fibroblast and the collagen fibrils." This result is supported by the TEM images in Fig. 2, which show fibrils interdigitating with the cell membrane. Because fibril orientation around the cell is not completely uniform, the fibrils will stretch independently, creating nonlinear cell strains and nonuniform cellular distortions as seen in Arnozczky et al. (3). The nonuniform fibril strain patterns seen must produce local cellular distortions far beyond what occurs on monolayers of cultured cells and must contribute to mechanically induced cell death reported herein. These large local distortions from nonuniform fibril loading, fibrils slipping past one another, and complex cell-matrix interactions help explain the results of this study where changes in cellular damage occur before the onset of structural damage. In contrast to the complex loadings and large distortions of fibroblast in vivo, fibroblasts in vitro on stretched membranes undergo less complex and less distortional loadings. Although in vitro stretched membrane cell deformation studies provide extremely valuable information about biological mechanisms, they may not accurately simulate in situ cells during ligament deformation.

Considering the organization of the fibroblast-ECM interaction, one may expect to see cellular damage in longitudinal lines along the axis of the ligament. However, it is reasonable that cellular damage is not seen in longitudinal lines in this study. By using the above techniques, slices through the tissue are compiled and overlaid, and because all fibers and fibrils are not perfectly aligned and do sit skewed from one another through the depth of the tissue one would not expect to see clear longitudinal distributions. By overlaying slices, the slight mismatching of collagen fibers or fibrils, size effects, and fibril interweaving and overlap limit the ability to see longitudinal columns because all the slices are "compressed" together. It is entirely possible that many semilongitudinal scans are adjacent, skewed, or slightly angled as a result of complex fibril organization, resulting in the distributions seen in Fig. 1. It should be noted that, because only regions of necrotic cells are being quantified (overlaid yellow regions are counted as damaged) and green (viable) regions are not being quantified, the overlaying process does not induce an error in the cellular damage mea- 
sure used in this study because cellular damage only considers necrotic cells normalized by tissue area.

Limitations exist in this study and should be noted. Structural damage was measured at only one rate of loading from a preloaded state, and the effect of multiple loadings was not investigated. In addition, the ligament microstructure was not examined for collagen fiber or fibril integrity (tearing or plastic deformation), and the mechanical properties were evaluated with only one tissue per applied subfailure stretch. Cellular damage was examined only at one time point and, therefore, only early cellular damage is quantified. In addition, processing times may have increased the magnitude of cell death in the tissue, yet changes in cellular damage are seen to increase with strain in tissues processed in the same controlled manner. The time-dependent aspects of cellular damage and the possibility of further necrosis resulting from apoptosis remain unexplored. In addition, in vivo loads in rat MCLs are not known and, hence, this behavior must be studied in a better model with more elucidation into the in vivo reference states regarding tissue microstructure and deformation during normal activity. The rat model used herein leaves questions open regarding effects of ligament size and species. Further studies in human ligaments are recommended.

In summary, structural and cellular damage occur at different levels of tissue strain in a rat MCL. Subfailure strain above the damage threshold changed the mechanical properties of the ligament. Further investigations into loading rate, multiple loadings, ligament microstructural displacements and deformations, cell deformation, and cell biology need to be performed to understand the subfailure behavior of ligament and the role of cell death in the healing process.

We thank Dr. Yan Lu for assistance with confocal microscopy, Dr. Tom Warner for evaluation of transmission electron microscopy, and Glen Leverson for computational assistance.

This work was thankfully funded in part by the National Science Foundation (Grant no. CMS-9907977), National Aeronautics and Space Administration (Grant no. NAG9-1152), and the University of Wisconsin-Madison Graduate School.

\section{REFERENCES}

1. Almekinders LC, Banes AJ, and Ballenger CA. Effects of repetitive motion on human fibroblasts. Med Sci Sports Exerc 25: 603-607, 1993.

2. Andriachi T, Sabiston P, De Haven K, Dahners L, Woo S, Frank C, Oakes B, Brand R, and Lewis J. Ligament: injury and repair. In: Injury and Repair of the Musculoskeletal Soft Tissues, edited by Woo SL-Y and Buckwalter JA. Park Ridge, IL: AAOS, 1987.

3. Arnoczky SP, Hoonjan A, Whallon B, and Cloutier B. Cell deformation in tendons under tensile load: a morphological analysis using confocal laser microscopy. Trans Orthop Res Soc 40: 495, 1994.

4. Blake RL, Anderson K, and Ferguson H. Posterior tibial tendinitis. A literature review with case reports. $J$ Am Podiatr Med Assoc 84: 141-149, 1994.

5. Buckwalter JA and Woo SLY. Effects of repetitive loading and motion on the musculoskeletal tissues. In: Orthopaedic Sports Medicine: Principles and Practice. Philadelphia, PA: Saunders, 1994.

6. Burr A, Hild F, and Leckie FA. Micro-mechanics and continuum damage mechanics. Arch Appl Mech 65: 437-456, 1995.
7. Chelikani S and Panjabi MM. Biomechanical symmetry of the rabbit ACL. Ann Mtg Am Soc Biomech Stanford, CA, 1995.

8. Curwin S and Stanish WD. Tendinitis: Its Etiology and Treatment. Lexington, MA: Collamore, 1984.

9. Daniel DM, Stone ML, Dobson BE, Fithian DC, Rossman DJ, and Kaufman KR. Fate of the ACL-injured patient. A prospective outcome study. Am J Sports Med 22: 632-644, 1994.

10. Fruensgaard $\mathbf{S}$ and Johannsen HV. Incomplete ruptures of the anterior cruciate ligament. J Bone Joint Surg Br 71: 526530, 1989.

11. Gilbert JA, Weinhold PS, Banes AJ, Link GW, and Jones GL. Strain profiles for circular cell culture plates containing flexible surfaces employed to mechanically deform cells in vitro. J Biomech 27: 1169-1177, 1994.

12. Gomez MA. The biomechanical properties of normal and healing ligaments. In: The Anterior Cruciate Ligament: Current and Future Concepts, edited by Arnoczky SP, Frank CB, Woo SL-Y, and Simon TM. New York: Raven, 1993, p. 227-233.

13. Gudi SR, Lee AA, Clark CB, and Frangos JA. Equibiaxial strain and strain rate stimulate early activation of $\mathrm{G}$ proteins in cardiac fibroblasts. Am J Physiol Cell Physiol 274: C1424C1428, 1998.

14. Hsieh AH, Tsai CM, Ma QJ, Lin T, Banes AJ, Villarreal FJ, Akeson WH, and Sung KL. Time-dependent increases in typeIII collagen gene expression in medical collateral ligament fibroblasts under cyclic strains. J Orthop Res 18: 220-227, 2000.

15. Hurschler C. Collagen Matrix in Normal and Healing Ligaments: Microstructural Behavior, Biological Adaptation and a Structural Mechanical Model (PhD thesis). Madison, WI: University of Wisconsin, 1998.

16. Hurschler C, Loitz-Ramage B, and Vanderby R Jr. A structurally based stress-stretch relationship for tendon and ligament. J Biomech Eng 119: 392-399, 1997.

17. Hurschler C, Provenzano PP, and Vanderby R Jr. Scanning electron microscopic investigation of healing and normal rat medial collateral ligaments fixed under slack and loaded conditions. Trans Orthop Res Soc 23: 1032, 1998.

18. Kastelic J, Galeski A, and Baer E. The multicomposite structure of tendon. Connect Tissue Res 6: 11-23, 1978.

19. Kennedy JC, Hawkins RJ, Willis RB, and Danylchuk KD. Tension studies of human knee ligaments. Yield point, ultimate failure, and disruption of the cruciate and tibial collateral ligaments. J Bone Joint Surg Am 58: 350-355, 1976.

20. Kiefhaber TR and Stern PJ. Upper extremity tendinitis and overuse syndromes in the athlete. Clin Sports Med 11: 39-55, 1992.

21. Krajcinovic D. Damage mechanics. Mech Matls 8: 117-197, 1989.

22. Kukreti U and Belkoff SM. Collagen fibril D-period may change as a function of strain and location in ligament. $J$ Biomech 33: 1569-1574, 2000.

23. Laws G and Walton M. Fibroblastic healing of grade II ligament injuries. Histological and mechanical studies in the sheep. J Bone Joint Surg Br 70: 390-396, 1988.

24. Lindgren BW. Statistical Theory. New York: Macmillan, 1976.

25. National Institutes of Health. NIH Image. http://rsb.info.nih. gov/nih-image, 2000.

26. Noyes FR, Butler DL, Grood ES, Zernicke RF, and Hefzy MS. Biomechanical analysis of human ligament grafts used in knee-ligament repairs and reconstructions. J Bone Joint Surg Am 66: 344-352, 1984.

27. Ohlendorf C, Tomford WW, and Mankin HJ. Chondrocyte survival in cryopreserved osteochondral articular cartilage. J Orthop Res 14: 413-416, 1996.

28. Panjabi MM, Yoldas E, Oxland TR, and Crisco JJ 3rd. Subfailure injury of the rabbit anterior cruciate ligament. $J$ Orthop Res 14: 216-222, 1996.

29. Provenzano PP, Hurschler C, and Vanderby R Jr. Microstructural morphology in the transition region between scar and intact residual segments of a healing rat medial collateral ligament. Connect Tissue Res 42: In Press, 2001.

30. Provenzano PP, Lakes RS, Keenan $\mathbf{T}$, and Vanderby $\mathbf{R}$ Jr. Non-linear ligament viscoelasticity. Ann Biomed Eng 29: 908-914, 2001. 
31. Raudino F. Radial sensory nerve entrapment in carpal tunnel syndrome. Electromyogr Clin Neurophysiol 35: 201-205, 1995.

32. Rigby BJ, Hirai N, Spikes JD, and Eyring H. The mechanical properties of rat tail tendon. J Gen Physiol 43: 265-289, 1959.

33. Sasaki N and Odajima W. Elongation mechanism of collagen fibrils and force-strain relations of tendon at each level of structural hierarchy. J Biomech 29: 1131-1136, 1996.

34. Sasaki N, Shukunami N, Matsushima N, and Izumi Y. Time-resolved X-ray diffraction from tendon collagen during creep using synchrotron radiation. J Biomech 32: 285-292, 1999.

35. Sims JA and Moorman SJ. The role of the iliolumbar ligament in low back pain. Med Hypotheses 45: 511-515, 1996.

36. Squier CA and Bausch WH. Three-dimensional organization of fibroblasts and collagen fibrils in rat tail tendon. Cell Tissue Res 238: 319-327, 1984
37. Turner S. Creep in glassy polymers. In: The Physics of Glassy Polymers, edited by RH Howard. New York: Wiley, 1973.

38. Viidik A. Simultaneous mechanical and light microscopic studies of collagen fibers. Z Anat Entwicklungsgesch 136: 204-212, 1972.

39. Wang VM, Bucchieri JS, Pollock RG, Flatow EL, Pawluk RJ, Bigliani LU, and Mow VC. The effects of cyclic loads on the subfailure properties of the inferior glenohumeral ligament. ASME Bioeng Conf 35: 137-138, 1997.

40. Winston FK, Macarak EJ, Gorfien SF, and Thibault LE. A system to reproduce and quantify the biomechanical environment of the cell. J Appl Physiol 67: 397-405, 1989.

41. Yahia L, Brunet J, Labelle S, and Rivard CH. A scanning electron microscopic study of rabbit ligaments under strain. Matrix 10: 58-64, 1990.

42. Yoganandan N, Maiman DJ, Pintar F, Ray G, Myklebust JB, Sances A Jr, and Larson SJ. Microtrauma in the lumbar spine: a cause of low back pain. Neurosurgery 23: 162-168.

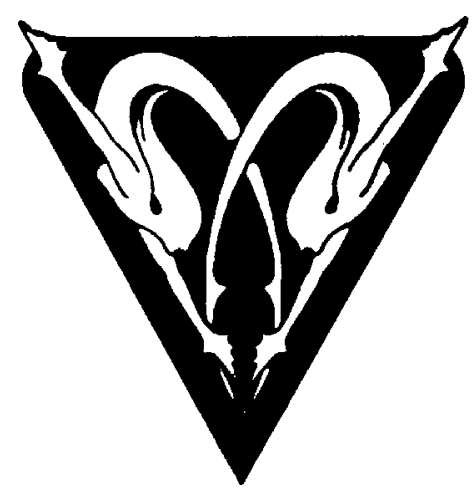

